\title{
Approximate Bayesian Computation
}

\author{
Mikael Sunnåker ${ }^{1,2 *}$, Alberto Giovanni Busetto ${ }^{2,39}$, Elina Numminen ${ }^{49}$, Jukka Corander ${ }^{4}$, Matthieu Foll ${ }^{2,5}$, \\ Christophe Dessimoz ${ }^{3,6,7 *}$
}

1 Department of Biosystems Science and Engineering, ETH Zurich, Zurich, Switzerland, 2 Competence Center for Systems Physiology and Metabolic Diseases, Zurich, Switzerland, 3 Department of Computer Science, ETH Zurich, Zurich, Switzerland, 4 Department of Mathematics and Statistics, University of Helsinki, Helsinki, Finland, 5 CMPG Institute of Ecology and Evolution, University of Bern, Bern, Switzerland, 6 Swiss Institute of Bioinformatics, Zurich, Switzerland, 7 EMBL-European Bioinformatics Institute, Cambridge, United Kingdom

\begin{abstract}
Approximate Bayesian computation (ABC) constitutes a class of computational methods rooted in Bayesian statistics. In all model-based statistical inference, the likelihood function is of central importance, since it expresses the probability of the observed data under a particular statistical model, and thus quantifies the support data lend to particular values of parameters and to choices among different models. For simple models, an analytical formula for the likelihood function can typically be derived. However, for more complex models, an analytical formula might be elusive or the likelihood function might be computationally very costly to evaluate. $A B C$ methods bypass the evaluation of the likelihood function. In this way, $A B C$ methods widen the realm of models for which statistical inference can be considered. $A B C$ methods are mathematically well-founded, but they inevitably make assumptions and approximations whose impact needs to be carefully assessed. Furthermore, the wider application domain of $A B C$ exacerbates the challenges of parameter estimation and model selection. $A B C$ has rapidly gained popularity over the last years and in particular for the analysis of complex problems arising in biological sciences (e.g., in population genetics, ecology, epidemiology, and systems biology).
\end{abstract}

This is a "Topic Page" article for PLOS Computational Biology.

\section{History}

The first Approximate Bayesian computation (ABC)-related ideas date back to the 1980s. Donald Rubin, when discussing the interpretation of Bayesian statements in 1984 [1], described a hypothetical sampling mechanism that yields a sample from the posterior distribution. This scheme was more of a conceptual thought experiment to demonstrate what type of manipulations are done when inferring the posterior distributions of parameters. The description of the sampling mechanism coincides exactly with that of the ABC-rejection scheme, and this article can be considered to be the first to describe approximate Bayesian computation. Another prescient point was made when Rubin argued that in Bayesian inference, applied statisticians should not settle for analytically tractable models only but instead consider computational methods that allow them to estimate the posterior distribution of interest. This way, a wider range of models can be considered. These arguments are particularly relevant in the context of ABC.

In 1984, Peter Diggle and Richard Gratton suggested using a systematic simulation scheme to approximate the likelihood function in situations where its analytic form is intractable [2]. Their method was based on defining a grid in the parameter space and using it to approximate the likelihood by running several simulations for each grid point. The approximation was then improved by applying smoothing techniques to the outcomes of the simulations. While the idea of using simulation for hypothesis testing was not new [3,4], Diggle and Gratton seemingly introduced the first procedure using simulation to do statistical inference under a circumstance where the likelihood is intractable.

Although Diggle and Gratton's approach had opened a new frontier, their method was not yet exactly identical to what is now known as ABC, as it aimed at approximating the likelihood rather than the posterior distribution. An article of Simon Tavaré et al. [5] was first to propose an $\mathrm{ABC}$ algorithm for posterior inference. In their seminal work, inference about the genealogy of DNA sequence data was considered, and in particular the problem of deciding the posterior distribution of the time to the most recent common ancestor of the sampled individuals. Such inference is analytically intractable for many demographical models, but the authors presented ways of simulating coalescent trees under the putative models. A sample from the posterior of model parameters was obtained by accepting/rejecting proposals based on comparing the number of segregating sites in the synthetic and real data. This work was followed by an applied study on modeling the variation in human $\mathrm{Y}$ chromosome by Jonathan K. Pritchard et al. [6] using the ABC method. Finally, the term Approximate Bayesian Computation was established by Mark Beaumont et al. [7], extending further the ABC methodology and discussing the suitability of the ABC-approach more specifically for problems in population genetics. Since then, ABC has spread to applications

Citation: Sunnåker $M$, Busetto $A G$, Numminen $E$, Corander $J$, Foll $M$, et al. (2013) Approximate Bayesian Computation. PLoS Comput Biol 9(1): e1002803. doi:10.1371/journal.pcbi.1002803

Editor: Shoshana Wodak, University of Toronto, Canada

Published January 10, 2013

Copyright: (c) 2013 Sunnåker et al. This is an open-access article distributed under the terms of the Creative Commons Attribution License, which permits unrestricted use, distribution, and reproduction in any medium, provided the original author and source are credited.

Funding: MS was supported by SystemsX.ch (RTD project YeastX). AGB was supported by SystemsX.ch (RTD projects YeastX and LiverX). JC was supported by the European Research Council grant no. 239784. EN was supported by the FICS graduate school. MF was supported by a Swiss NSF grant No 3100-126074 to Laurent Excoffier. CD was supported by SNSF advanced researcher fellowship no. 136461. The funders had no role in the preparation of the manuscript.

Competing Interests: The authors have declared that no competing interests exist.

* E-mail: mikael.sunnaker@bsse.ethz.ch (MS); dessimoz@ebi.ac.uk (CD)

9 These authors contributed equally to this work. 
outside population genetics, such as systems biology, epidemiology, or phylogeography.

\section{Method}

\section{Motivation}

A common incarnation of the Bayes' theorem relates the conditional probability (or density) of a particular parameter value $\theta$ given data $D$ to the probability of $D$ given $\theta$ by the rule:

$$
p(\theta \mid D)=\frac{p(D \mid \theta) p(\theta)}{p(D)}
$$

where $p(\theta \mid D)$ denotes the posterior, $p(D \mid \theta)$ the likelihood, $p(\theta)$ the prior, and $p(D)$ the evidence (also referred to as the marginal likelihood or the prior predictive probability of the data).

The prior represents beliefs about $\theta$ before $D$ is available, and it is often specified by choosing a particular distribution among a set of well-known and tractable families of distributions, such that both the evaluation of prior probabilities and random generation of values of $\theta$ are relatively straightforward. For certain kinds of models, it is more pragmatic to specify the prior $p(\theta)$ using a factorization of the joint distribution of all the elements of $\theta$ in terms of a sequence of their conditional distributions. If one is only interested in the relative posterior plausibilities of different values of $\theta$, the evidence $p(D)$ can be ignored, as it constitutes a normalising constant, which cancels for any ratio of posterior probabilities. It remains, however, necessary to evaluate the likelihood $p(D \mid \theta)$ and the prior $p(\theta)$. For numerous applications, it is computationally expensive, or even completely infeasible, to evaluate the likelihood [8], which motivates the use of ABC to circumvent this issue.

\section{The $A B C$ Rejection Algorithm}

All ABC-based methods approximate the likelihood function by simulations, the outcomes of which are compared with the observed data [9-11]. More specifically, with the $\mathrm{ABC}$ rejection algorithm - the most basic form of $\mathrm{ABC}$ - a set of parameter points is first sampled from the prior distribution. Given a sampled parameter point $\theta$, a dataset $\hat{D}$ is then simulated under the statistical model $M$ specified by $\theta$. If the generated $\hat{D}$ is too different from the observed data $D$, the sampled parameter value is discarded. In precise terms, $\hat{D}$ is accepted with tolerance $\varepsilon \geq 0$ if:

$$
\rho(\hat{D}, D) \leq \varepsilon,
$$

where the distance measure $\rho(\hat{D}, D)$ determines the level of discrepancy between $\hat{D}$ and $D$ based on a given metric (e.g., the Euclidean distance). A strictly positive tolerance is usually necessary, since the probability that the simulation outcome coincides exactly with the data (event $\hat{D}=D$ ) is negligible for all but trivial applications of $\mathrm{ABC}$, which would in practice lead to rejection of nearly all sampled parameter points. The outcome of the $\mathrm{ABC}$ rejection algorithm is a sample of parameter values approximately distributed according to the desired posterior distribution and, crucially, obtained without the need of explicitly evaluating the likelihood function (Figure 1).

\section{Summary Statistics}

The probability of generating a dataset $\hat{D}$ with a small distance to $D$ typically decreases as the dimensionality of the data increases. This leads to a substantial decrease in the computational efficiency of the above basic $\mathrm{ABC}$ rejection algorithm. A common approach to lessen this problem is to replace $D$ with a set of lower dimensional summary statistics $S(D)$, which are selected to capture the relevant information in $D$. The acceptance criterion in $\mathrm{ABC}$ rejection algorithm becomes:

$$
\rho(S(\hat{D}), S(D)) \leq \varepsilon
$$

If the summary statistics are sufficient with respect to the model parameters $\theta$, the efficiency increase obtained in this way does not introduce any error [12]. Indeed, by definition, sufficiency implies that all information in $D$ about $\theta$ is captured by $S(D)$.

As elaborated below, it is typically impossible, outside the exponential family of distributions, to identify a finite-dimensional set of sufficient statistics. Nevertheless, informative, but possibly nonsufficient, summary statistics are often used in applications where inference is performed with $\mathrm{ABC}$ methods.

\section{Example}

An illustrative example is a bistable system that can be characterized by a hidden Markov model (HMM) subject to measurement noise (Figure 2). Such models are employed for many biological systems: they have for example been used in Development, signaling, activation/deactivation, logical processing, and non-equilibrium thermodynamics. For instance, the behavior of the Sonic Hedgehog (Shh) transcription factor in Drosophila melanogaster can be modeled with a HMM [13]. The (biological) dynamical model consists of two states: $A$ and $B$. If the probability of a transition from one state to the other is defined as $\theta$ in both directions, the probability to remain in the same state at each time step is $1-\theta$. The probability to measure the state correctly is $\gamma$ (conversely, the probability of an incorrect measurement is $1-\gamma$ ).

Due to the conditional dependencies between states at different time points, calculation of the likelihood of time series data is somewhat tedious, which illustrates the motivation to use ABC. A computational issue for the basic $\mathrm{ABC}$ is the large dimensionality of the data in an application like this. This can be reduced using the summary statistic $S$, which is the frequency of switches between the two states. As a distance measure $\rho(\cdot, \cdot)$, the absolute difference is used, combined with a tolerance $\varepsilon=2$. The posterior inference about the parameter $\theta$ can be done following the five steps presented in Figure 1:

Step 1: Assume that the observed data are the state sequence AAAABAABBAAAAAABAAAA, which was generated using $\theta=0.25$. The associated summary statistic, the number of switches between the states in the experimental data, is $\omega_{E}=6$.

Step 2: Assuming nothing is known about $\theta$, a uniform prior in the interval $[0,1]$ is employed. A number $n$ of parameter points are drawn from the prior, and the model is simulated for each of the parameter points $\theta_{i}, i=1, \ldots, n$, which results in $n$ sequences of simulated data. In this example, $n=5$, with each drawn parameter and simulated dataset recorded in Table 1, column 2-3. In practice, $n$ would need to be much larger to obtain an appropriate approximation.

Step 3: The summary statistic is being computed for each sequence of simulated data, $\omega_{S, i}, i=1, \ldots, n$ (Table 1 , column 4).

Step 4: The distance between the observed and simulated transition frequencies $\rho\left(\omega_{S, i}, \omega_{E}\right)=\left|\omega_{S, i}-\omega_{E}\right|$ is computed for all parameter points (Table 1, column 5). Parameter points for which the distance is smaller than or equal to $\varepsilon$ are accepted as approximate samples from the posterior (Table 1, column 6). 


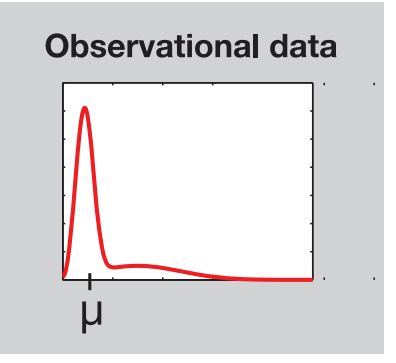

(1) Compute summary statistic $\mu$ from observational data
(3) Compute summary statistic $\mu_{i}$ for each simulation

$$
\rho\left(\mu_{i}, \mu\right) \stackrel{?}{\leq} \varepsilon
$$

(4) Based on a distance $\rho(\cdot, \cdot)$ and a tolerance $\varepsilon$, decide for each simulation whether its summary statistic is sufficiently close to that of the observed data.
Prior distribution of model parameter $\theta$
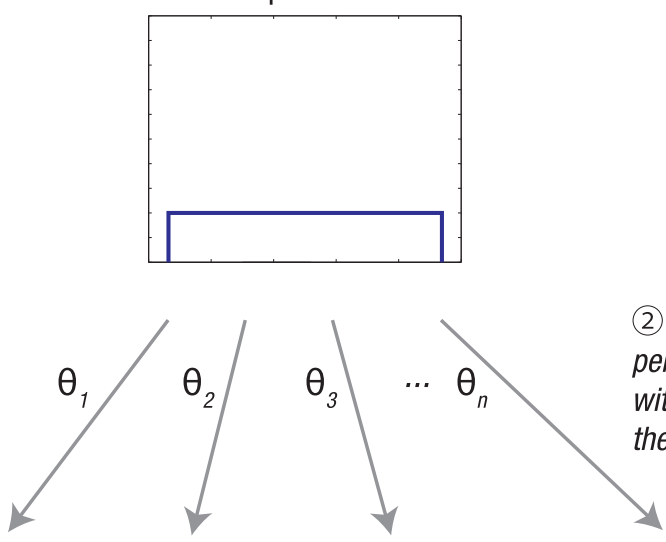

(2) Given a certain model, perform $n$ simulations, each with a parameter drawn from the prior distribution
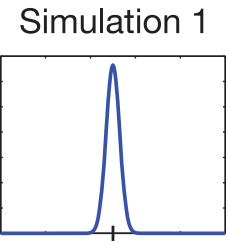

$\mu_{1}$
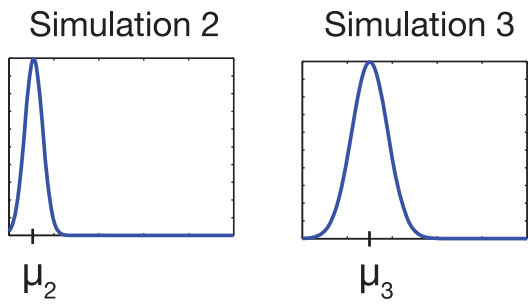

\section{Simulation $\mathrm{n}$}

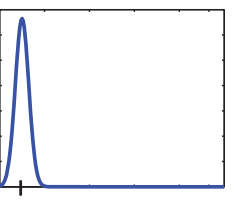

$\mu_{\mathrm{n}}$

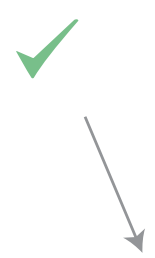

Posterior distribution of

(5) Approximate the posterior model parameter $\theta$ distribution of $\theta$ from the distribution

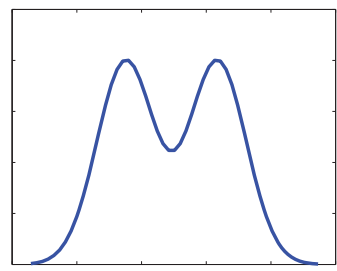

of parameter values $\theta_{i}$ associated with accepted simulations.

Figure 1. Parameter estimation by Approximate Bayesian Computation: a conceptual overview. doi:10.1371/journal.pcbi.1002803.g001

Step 5: The posterior distribution is approximated with the accepted parameter points. The posterior distribution should have a nonnegligible probability for parameter values in a region around the true value of $\theta$ in the system, if the data are sufficiently informative. In this example, the posterior probability mass is evenly split between the values 0.08 and 0.43 .

Figure 3 shows the posterior probabilities obtained by $\mathrm{ABC}$ and a large $n$ using either the summary statistic combined with $(\varepsilon=0$ and $\varepsilon=2$ ) or the full data sequence. These are compared with the true posterior, which can be computed exactly and efficiently using the Viterbi algorithm. The used summary statistic is not sufficient, and it is seen that even with $\varepsilon=0$, the deviation from the theoretical posterior is considerable. Of note, a much longer observed data sequence would be required to obtain a posterior that is concentrated around the true value of $\theta(\theta=0.25)$.
This example application of $\mathrm{ABC}$ used simplifications for illustrative purposes. A number of review articles provide pointers to more realistic applications of ABC [9-11,14].

\section{Model Comparison with ABC}

Besides parameter estimation, the ABC-framework can be used to compute the posterior probabilities of different candidate models [15-17]. In such applications, one possibility is to use the rejection-sampling in a hierarchical manner. First, a model is sampled from the prior distribution for the models; then, given the model sampled, the model parameters are sampled from the prior distribution assigned to that model. Finally, a simulation is performed as in the single-model ABC. The relative acceptance frequencies for the different models now approximate the posterior 


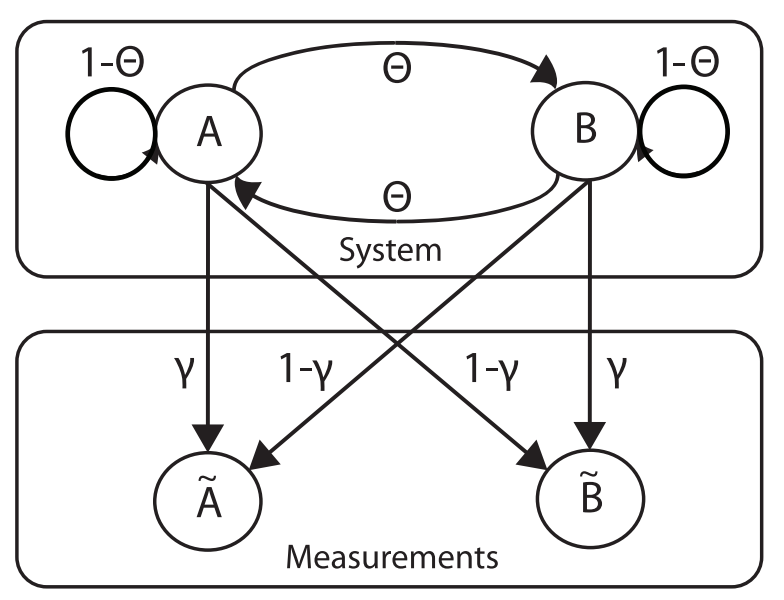

Figure 2. A dynamic bistable hidden Markov model. doi:10.1371/journal.pcbi.1002803.g002

distribution for these models. Again, computational improvements for $\mathrm{ABC}$ in the space of models have been proposed, such as constructing a particle filter in the joint space of models and parameters [17].

Once the posterior probabilities of models have been estimated, one can make full use of the techniques of Bayesian model comparison. For instance, to compare the relative plausibilities of two models $M_{1}$ and $M_{2}$, one can compute their posterior ratio, which is related to the Bayes factor $B_{1,2}$ :

$$
\frac{p\left(M_{1} \mid D\right)}{p\left(M_{2} \mid D\right)}=\frac{p\left(D \mid M_{1}\right)}{p\left(D \mid M_{2}\right)} \frac{p\left(M_{1}\right)}{p\left(M_{2}\right)}=B_{1,2} \frac{p\left(M_{1}\right)}{p\left(M_{2}\right)} .
$$

If the model priors are equal $\left(p\left(M_{1}\right)=p\left(M_{2}\right)\right)$, the Bayes factor equals the posterior ratio.

In practice, as discussed below, these measures can be highly sensitive to the choice of parameter prior distributions and summary statistics, and thus conclusions of model comparison should be drawn with caution.

\section{Pitfalls and Remedies}

As for all statistical methods, a number of assumptions and approximations are inherently required for the application of ABC-based methods to real modeling problems. For example, setting the tolerance parameter $\varepsilon$ to zero ensures an exact result but typically makes computations prohibitively expensive. Thus, values of $\varepsilon$ larger than zero are used in practice, which introduces a bias. Likewise, sufficient statistics are typically not available, and instead, other summary statistics are used, which introduces an additional bias due to the loss of information. Additional sources of bias - for example, in the context of model selection - may be more subtle $[12,18]$.

At the same time, some of the criticisms that have been directed at the $\mathrm{ABC}$ methods, in particular within the field of phylogeography [19-21], are not specific to $A B C$ and apply to all Bayesian methods or even all statistical methods (e.g., the choice of prior distribution and parameter ranges) [9,22]. However, because of the ability of ABC-methods to handle much more complex models, some of these general pitfalls are of particular relevance in the context of $\mathrm{ABC}$ analyses.

This section discusses these potential risks and reviews possible ways to address them (Table 2).

\section{Approximation of the Posterior}

A nonnegligible $\varepsilon$ comes with the price that one samples from $p(\theta \mid \rho(\hat{D}, D) \leq \varepsilon)$ instead of the true posterior $p(\theta \mid D)$. With a sufficiently small tolerance, and a sensible distance measure, the resulting distribution $p(\theta \mid \rho(\hat{D}, D) \leq \varepsilon)$ should often approximate the actual target distribution $p(\theta \mid D)$ reasonably well. On the other hand, a tolerance that is large enough that every point in the parameter space becomes accepted will yield a replica of the prior distribution. There are empirical studies of the difference between $p(\theta \mid \rho(\hat{D}, D) \leq \varepsilon)$ and $p(\theta \mid D)$ as a function of $\varepsilon$ [23], and theoretical results for an upper $\varepsilon$-dependent bound for the error in parameter estimates [24]. The accuracy of the posterior (defined as the expected quadratic loss) delivered by $\mathrm{ABC}$ as a function of $\varepsilon$ has also been investigated [25]. However, the convergence of the distributions when $\varepsilon$ approaches zero, and how it depends on the distance measure used, is an important topic that has yet to be investigated in greater detail. In particular, it remains difficult to disentangle errors introduced by this approximation from errors due to model mis-specification [9].

As an attempt to correct some of the error due to a non-zero $\varepsilon$, the usage of local linear weighted regression with $\mathrm{ABC}$ to reduce the variance of the posterior estimates has been suggested [7]. The method assigns weights to the parameters according to how well simulated summaries adhere to the observed ones and performs linear regression between the summaries and the weighted parameters in the vicinity of observed summaries. The obtained regression coefficients are used to correct sampled parameters in the direction of observed summaries. An improvement was suggested in the form of nonlinear regression using a feed-forward neural network model [26]. However, it has been shown that the posterior distributions obtained with these approaches are not always consistent with the prior distribution, which did lead to a reformulation of the regression adjustment that respects the prior distribution [27].

Finally, statistical inference using $\mathrm{ABC}$ with a non-zero tolerance $\varepsilon$ is not inherently flawed: under the assumption of

Table 1. Example of $A B C$ rejection algorithm.

\begin{tabular}{|c|c|c|c|c|c|}
\hline $\mathbf{i}$ & $\boldsymbol{\theta}_{i}$ & Simulated Datasets (Step 2) & Summary Statistic $\omega_{s, i}($ Step 3$)$ & Distance $\rho\left(\omega_{S, i i} \omega_{E}\right)($ Step 4) & Outcome (Step 4) \\
\hline 1 & 0.08 & AABAAAABAABAAABAAAAA & 8 & 2 & accepted \\
\hline 2 & 0.68 & AABBABABAAABBABABBAB & 13 & 7 & rejected \\
\hline 3 & 0.87 & BBВABВАВBВBABABBBBBA & 9 & 3 & rejected \\
\hline 4 & 0.43 & AABAAAAABBABBBBBBBBA & 6 & 0 & accepted \\
\hline 5 & 0.53 & ABBBBBAABBABBABAABBB & 9 & 3 & rejected \\
\hline
\end{tabular}




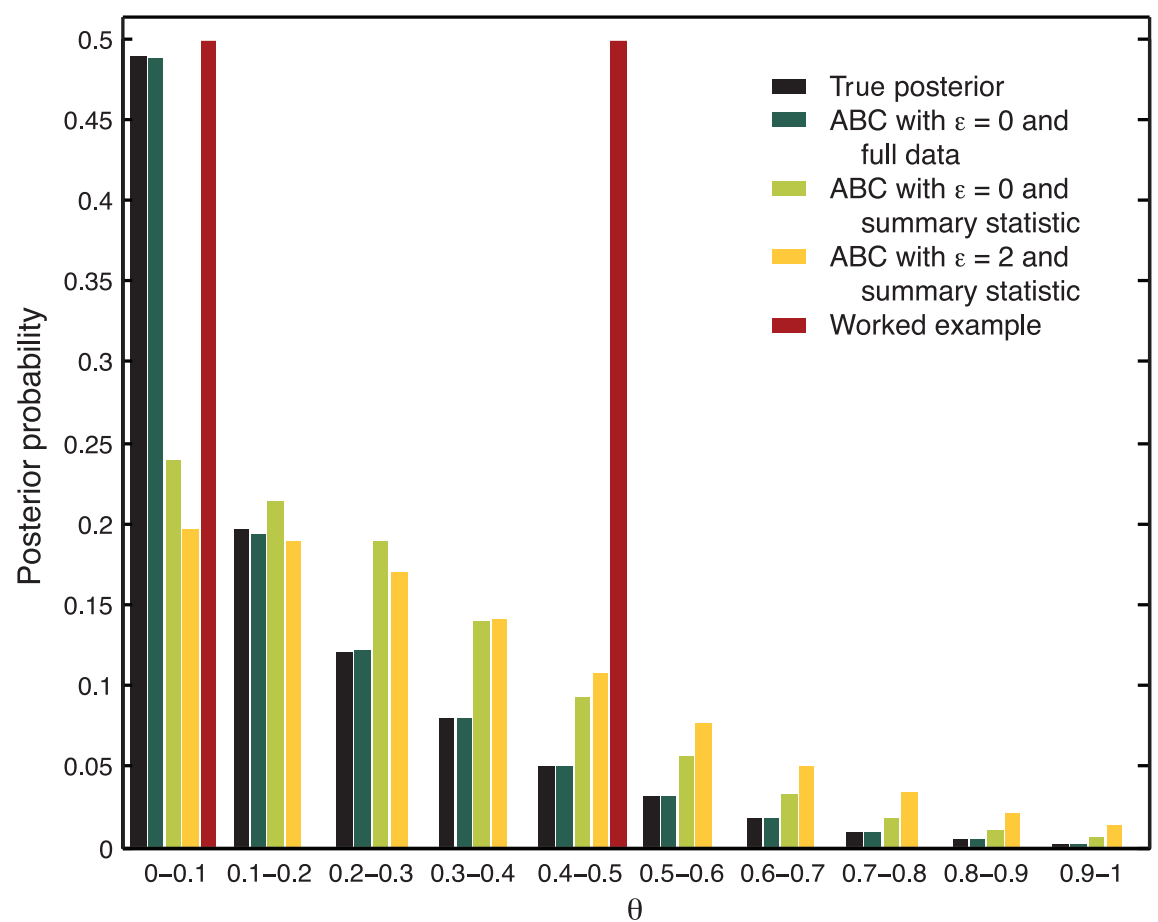

Figure 3. Posterior of $\theta$ obtained in the example (red), compared with the true posterior distribution (black), and ABC simulations with large $n$. The use of the insufficient summary statistic $\omega$ introduces a bias, even when requiring $\varepsilon=0$ (light green). doi:10.1371/journal.pcbi.1002803.g003

measurement errors, the optimal $\varepsilon$ can in fact be shown to be not zero $[25,28]$. Indeed, the bias caused by a non-zero tolerance can be characterized and compensated by introducing a specific form of noise to the summary statistics. Asymptotic consistency for such "noisy $\mathrm{ABC}$ " has been established, together with formulas for the asymptotic variance of the parameter estimates for a fixed tolerance [25].

Table 2. Potential risks and remedies in $A B C$-based statistical inference.

\begin{tabular}{|c|c|c|c|}
\hline Error Source & Potential Issue & Solution & Subsection \\
\hline Nonzero tolerance $\varepsilon$ & $\begin{array}{l}\text { The inexactness introduces a bias in } \\
\text { the computed posterior distribution. }\end{array}$ & $\begin{array}{l}\text { Theoretical/practical studies of the } \\
\text { sensitivity of the posterior distribution to } \\
\text { the tolerance. Noisy } A B C \text {. }\end{array}$ & Approximation of the posterior \\
\hline Nonsufficient summary statistics & $\begin{array}{l}\text { The information loss causes inflated } \\
\text { credible intervals. }\end{array}$ & $\begin{array}{l}\text { Automatic selection/semi-automatic } \\
\text { identification of sufficient statistics. } \\
\text { Model validation checks (e.g., } \\
\text { Templeton } 2009 \text { [19]). }\end{array}$ & $\begin{array}{l}\text { Choice and sufficiency of summary } \\
\text { statistics }\end{array}$ \\
\hline $\begin{array}{l}\text { Small number of models/mis- } \\
\text { specified models }\end{array}$ & $\begin{array}{l}\text { The investigated models are not } \\
\text { representative/lack predictive power. }\end{array}$ & $\begin{array}{l}\text { Careful selection of models. Evaluation } \\
\text { of the predictive power. }\end{array}$ & Small number of models \\
\hline Priors and parameter ranges & $\begin{array}{l}\text { Conclusions may be sensitive to the } \\
\text { choice of priors. Model choice may } \\
\text { be meaningless. }\end{array}$ & $\begin{array}{l}\text { Check sensitivity of Bayes factors to the } \\
\text { choice of priors. Some theoretical results } \\
\text { regarding choice of priors are available. } \\
\text { Use alternative methods for model } \\
\text { validation. }\end{array}$ & Prior distribution and parameter ranges \\
\hline Curse-of-dimensionality & $\begin{array}{l}\text { Low parameter acceptance rates. } \\
\text { Model errors cannot be distinguished } \\
\text { from an insufficient exploration of the } \\
\text { parameter space. Risk of overfitting. }\end{array}$ & $\begin{array}{l}\text { Methods for model reduction if } \\
\text { applicable. Methods to speed up the } \\
\text { parameter exploration. Quality } \\
\text { controls to detect overfitting. }\end{array}$ & Curse-of-dimensionality \\
\hline Model ranking with summary statistics & $\begin{array}{l}\text { The computation of Bayes factors on } \\
\text { summary statistics may not be related } \\
\text { to the Bayes factors on the original } \\
\text { data, which may therefore render the } \\
\text { results meaningless. }\end{array}$ & $\begin{array}{l}\text { Only use summary statistics that fulfill the } \\
\text { necessary and sufficient conditions to } \\
\text { produce a consistent Bayesian model } \\
\text { choice. Use alternative methods for } \\
\text { model validation. }\end{array}$ & $\begin{array}{l}\text { Bayes factor with } A B C \text { and summary } \\
\text { statistics }\end{array}$ \\
\hline Implementation & $\begin{array}{l}\text { Low protection to common } \\
\text { assumptions in the simulation } \\
\text { and the inference process. }\end{array}$ & $\begin{array}{l}\text { Sanity checks of results. Standardization } \\
\text { of software. }\end{array}$ & Indispensable quality controls \\
\hline
\end{tabular}

doi:10.1371/journal.pcbi.1002803.t002 
Table 3. Software incorporating $A B C$.

\begin{tabular}{|c|c|c|}
\hline Software & Keywords and Features & Reference \\
\hline DIY-ABC & $\begin{array}{l}\text { Software for fit of genetic data to complex situations. Comparison of competing models. Parameter estimation. } \\
\text { Computation of bias and precision measures for a given model and known parameters values. }\end{array}$ & [53] \\
\hline$A B C R$ package & $\begin{array}{l}\text { Several } A B C \text { algorithms for performing parameter estimation and model selection. Nonlinear heteroscedastic regression } \\
\text { methods for } A B C \text {. Cross-validation tool. }\end{array}$ & [54] \\
\hline ABC-SysBio & $\begin{array}{l}\text { Python package. Parameter inference and model selection for dynamical systems. Combines } A B C \text { rejection sampler, } A B C \\
\text { SMC for parameter inference, and } A B C \text { SMC for model selection. Compatible with models written in Systems Biology } \\
\text { Markup Language (SBML). Deterministic and stochastic models. }\end{array}$ & [55] \\
\hline ABCtoolbox & $\begin{array}{l}\text { Open source programs for various } A B C \text { algorithms including rejection sampling, MCMC without likelihood, a particle-based } \\
\text { sampler, and } A B C-G L M \text {. Compatibility with most simulation and summary statistics computation programs. }\end{array}$ & [56] \\
\hline msBayes & $\begin{array}{l}\text { Open source software package consisting of several } \mathrm{C} \text { and } \mathrm{R} \text { programs that are run with a Perl "front-end." Hierarchical } \\
\text { coalescent models. Population genetic data from multiple co-distributed species. }\end{array}$ & [57] \\
\hline PopABC & Software package for inference of the pattern of demographic divergence. Coalescent simulation. Bayesian model choice. & [58] \\
\hline ONeSAMP & $\begin{array}{l}\text { Web-based program to estimate the effective population size from a sample of microsatellite genotypes. Estimates of } \\
\text { effective population size, together with } 95 \% \text { credible limits. }\end{array}$ & [59] \\
\hline$A B C 4 F$ & Software for estimation of F-statistics for dominant data. & {$[60]$} \\
\hline 2BAD & $\begin{array}{l}\text { Two-event Bayesian ADmixture. Software allowing up to two independent admixture events with up to three parental } \\
\text { populations. Estimation of several parameters (admixture, effective sizes, etc.). Comparison of pairs of admixture models. }\end{array}$ & [61] \\
\hline
\end{tabular}

\section{Choice and Sufficiency of Summary Statistics}

Summary statistics may be used to increase the acceptance rate of $\mathrm{ABC}$ for high-dimensional data. Low-dimensional sufficient statistics are optimal for this purpose, as they capture all relevant information present in the data in the simplest possible form [11]. However, low-dimensional sufficient statistics are typically unattainable for statistical models where ABC-based inference is most relevant, and consequently, some heuristic is usually necessary to identify useful low-dimensional summary statistics. The use of a set of poorly chosen summary statistics will often lead to inflated credible intervals due to the implied loss of information [11], which can also bias the discrimination between models. A review of methods for choosing summary statistics is available [29], which may provide valuable guidance in practice.

One approach to capture most of the information present in data would be to use many statistics, but the accuracy and stability of ABC appears to decrease rapidly with an increasing numbers of summary statistics $[9,11]$. Instead, a better strategy is to focus on the relevant statistics only-relevancy depending on the whole inference problem, on the model used, and on the data at hand [30].

An algorithm has been proposed for identifying a representative subset of summary statistics, by iteratively assessing whether an additional statistic introduces a meaningful modification of the posterior [31]. One of the challenges here is that a large ABC approximation error may heavily influence the conclusions about the usefulness of a statistic at any stage of the procedure. Another method decomposes into two main steps [30]. First, a reference approximation of the posterior is constructed by minimizing the entropy. Sets of candidate summaries are then evaluated by comparing the ABC-approximated posteriors with the reference posterior.

With both of these strategies, a subset of statistics is selected from a large set of candidate statistics. Instead, the partial least squares regression approach uses information from all the candidate statistics, each being weighted appropriately [32]. Recently, a method for constructing summaries in a semiautomatic manner has attained a considerable interest [25]. This method is based on the observation that the optimal choice of summary statistics, when minimizing the quadratic loss of the parameter point estimates, can be obtained through the posterior mean of the parameters, which is approximated by performing a linear regression based on the simulated data.

Methods for the identification of summary statistics that could also simultaneously assess the influence on the approximation of the posterior would be of substantial value [33]. This is because the choice of summary statistics and the choice of tolerance constitute two sources of error in the resulting posterior distribution. These errors may corrupt the ranking of models and may also lead to incorrect model predictions. Indeed, none of the methods above assess the choice of summaries for the purpose of model selection.

\section{Bayes Factor with $A B C$ and Summary Statistics}

It has been shown that the combination of insufficient summary statistics and ABC for model selection can be problematic [12,18]. Indeed, if one lets the Bayes factor based on the summary statistic $S(D)$ be denoted by $B_{1,2}^{s}$, the relation between $B_{1,2}$ and $B_{1,2}^{s}$ takes the form [12]:

$B_{1,2}=\frac{p\left(D \mid M_{1}\right)}{p\left(D \mid M_{2}\right)}=\frac{p\left(D \mid S(D), M_{1}\right)}{p\left(D \mid S(D), M_{2}\right)} \frac{p\left(S(D) \mid M_{1}\right)}{p\left(S(D) \mid M_{2}\right)}=\frac{p\left(D \mid S(D), M_{1}\right)}{p\left(D \mid S(D), M_{2}\right)} B_{1,2}^{s}$.

Thus, a summary statistic $S(D)$ is sufficient for comparing two models $M_{1}$ and $M_{2}$ if and only if:

$$
p\left(D \mid S(D), M_{1}\right)=p\left(D \mid S(D), M_{2}\right),
$$

which results in that $B_{1,2}=B_{1,2}^{s}$. It is also clear from the equation above that there might be a huge difference between $B_{1,2}$ and $B_{1,2}^{s}$ if the condition is not satisfied, as can be demonstrated by toy examples $[12,16,18]$. Crucially, it was shown that sufficiency for $M_{1}$ or $M_{2}$ alone, or for both models, does not guarantee sufficiency for ranking the models [12]. However, it was also shown that any sufficient summary statistic for a model $M$ in which both $M_{1}$ and $M_{2}$ are nested is valid for ranking the nested models [12]. 
The computation of Bayes factors on $S(D)$ may therefore be misleading for model selection purposes, unless the ratio between the Bayes factors on $D$ and $S(D)$ would be available, or at least could be approximated reasonably well. Alternatively, necessary and sufficient conditions on summary statistics for a consistent Bayesian model choice have recently been derived [34], which can provide useful guidance.

However, this issue is only relevant for model selection when the dimension of the data has been reduced. ABC-based inference, in which the actual datasets are directly compared - as is the case for some systems biology applications (e.g., see [35]) - circumvents this problem.

\section{Indispensable Quality Controls}

As the above discussion makes clear, any $\mathrm{ABC}$ analysis requires choices and tradeoffs that can have a considerable impact on its outcomes. Specifically, the choice of competing models/hypotheses, the number of simulations, the choice of summary statistics, or the acceptance threshold cannot currently be based on general rules, but the effect of these choices should be evaluated and tested in each study [10].

A number of heuristic approaches to the quality control of ABC have been proposed, such as the quantification of the fraction of parameter variance explained by the summary statistics [10]. A common class of methods aims at assessing whether or not the inference yields valid results, regardless of the actually observed data. For instance, given a set of parameter values, which are typically drawn from the prior or the posterior distributions for a model, one can generate a large number of artificial datasets. In this way, the quality and robustness of $\mathrm{ABC}$ inference can be assessed in a controlled setting, by gauging how well the chosen $\mathrm{ABC}$ inference method recovers the true parameter values, and also models if multiple structurally different models are considered simultaneously.

Another class of methods assesses whether the inference was successful in light of the given observed data, for example by comparing the posterior predictive distribution of summary statistics to the summary statistics observed [10]. Beyond that, cross-validation techniques [36] and predictive checks $[37,38]$ represent promising future strategies to evaluate the stability and out-of-sample predictive validity of $\mathrm{ABC}$ inferences. This is particularly important when modeling large datasets, because then the posterior support of a particular model can appear overwhelmingly conclusive, even if all proposed models in fact are poor representations of the stochastic system underlying the observation data. Out-of-sample predictive checks can reveal potential systematic biases within a model and provide clues on to how to improve its structure or parametrization.

Interestingly, fundamentally novel approaches for model choice that incorporate quality control as an integral step in the process have recently been proposed. $\mathrm{ABC}$ allows, by construction, estimation of the discrepancies between the observed data and the model predictions, with respect to a comprehensive set of statistics. These statistics are not necessarily the same as those used in the acceptance criterion. The resulting discrepancy distributions have been used for selecting models that are in agreement with many aspects of the data simultaneously [39], and model inconsistency is detected from conflicting and codependent summaries. Another quality-control-based method for model selection employs $\mathrm{ABC}$ to approximate the effective number of model parameters and the deviance of the posterior predictive distributions of summaries and parameters [40]. The deviance information criterion is then used as measure of model fit. It has also been shown that the models preferred based on this criterion can conflict with those supported by Bayes factors. For this reason, it is useful to combine different methods for model selection to obtain correct conclusions.

Quality controls are achievable and indeed performed in many ABC-based works, but for certain problems, the assessment of the impact of the method-related parameters can be challenging. However, the rapidly increasing use of $\mathrm{ABC}$ can be expected to provide a more thorough understanding of the limitations and applicability of the method.

\section{General Risks in Statistical Inference Exacerbated in ABC}

This section reviews risks that are strictly speaking not specific to $\mathrm{ABC}$, but also relevant for other statistical methods as well. However, the flexibility offered by ABC to analyze very complex models makes them highly relevant to discuss here.

Prior distribution and parameter ranges. The specification of the range and the prior distribution of parameters strongly benefits from previous knowledge about the properties of the system. One criticism has been that in some studies the "parameter ranges and distributions are only guessed based upon the subjective opinion of the investigators" [41], which is connected to classical objections of Bayesian approaches [42].

With any computational method, it is typically necessary to constrain the investigated parameter ranges. The parameter ranges should if possible be defined based on known properties of the studied system but may for practical applications necessitate an educated guess. However, theoretical results regarding objective priors are available, which may for example be based on the principle of indifference or the principle of maximum entropy $[43,44]$. On the other hand, automated or semiautomated methods for choosing a prior distribution often yield improper densities. As most $\mathrm{ABC}$ procedures require generating samples from the prior, improper priors are not directly applicable to $\mathrm{ABC}$.

One should also keep the purpose of the analysis in mind when choosing the prior distribution. In principle, uninformative and flat priors that exaggerate our subjective ignorance about the parameters may still yield reasonable parameter estimates. However, Bayes factors are highly sensitive to the prior distribution of parameters. Conclusions on model choice based on Bayes factor can be misleading unless the sensitivity of conclusions to the choice of priors is carefully considered.

Small number of models. Model-based methods have been criticized for not exhaustively covering the hypothesis space [21]. Indeed, model-based studies often revolve around a small number of models, and due to the high computational cost to evaluate a single model in some instances, it may then be difficult to cover a large part of the hypothesis space.

An upper limit to the number of considered candidate models is typically set by the substantial effort required to define the models and to choose between many alternative options [10]. There is no commonly accepted ABC-specific procedure for model construction, so experience and prior knowledge are used instead [11]. Although more robust procedures for a priori model choice and formulation would be beneficial, there is no one-size-fits-all strategy for model development in statistics: sensible characterization of complex systems will always necessitate a great deal of detective work and use of expert knowledge from the problem domain.

Some opponents of ABC contend that since only few modelssubjectively chosen and probably all wrong - can be realistically considered, ABC analyses provide only limited insight [21]. However, there is an important distinction between identifying a plausible null hypothesis and assessing the relative fit of alternative 
hypotheses [9]. Since useful null hypotheses, that potentially hold true, can extremely seldom be put forward in the context of complex models, predictive ability of statistical models as explanations of complex phenomena is far more important than the test of a statistical null hypothesis in this context. It is also common to average over the investigated models, weighted based on their relative plausibility, to infer model features (e.g., parameter values) and to make predictions.

Large datasets. Large datasets may constitute a computational bottleneck for model-based methods. It was, for example, pointed out that in some ABC-based analyses, part of the data have to be omitted [21]. A number of authors have argued that large datasets are not a practical limitation [10,42], although the severity of this issue depends strongly on the characteristics of the models. Several aspects of a modeling problem can contribute to the computational complexity, such as the sample size, number of observed variables or features, time or spatial resolution, etc. However, with increasing computing power, this issue will potentially be less important.

Instead of sampling parameters for each simulation from the prior, it has been proposed alternatively to combine the Metropolis-Hastings algorithm with $\mathrm{ABC}$, which was reported to result in a higher acceptance rate than for plain ABC [33]. Naturally, such an approach inherits the general burdens of MCMC methods, such as the difficulty to assess convergence, correlation among the samples from the posterior [23], and relatively poor parallelizability [10].

Likewise, the ideas of sequential Monte Carlo (SMC) and population Monte Carlo (PMC) methods have been adapted to the ABC setting $[23,45]$. The general idea is to iteratively approach the posterior from the prior through a sequence of target distributions. An advantage of such methods, compared to ABC-MCMC, is that the samples from the resulting posterior are independent. In addition, with sequential methods the tolerance levels must not be specified prior to the analysis, but are adjusted adaptively [46].

It is relatively straightforward to parallelize a number of steps in ABC algorithms based on rejection sampling and sequential Monte Carlo methods. It has also been demonstrated that parallel algorithms may yield significant speedups for MCMC-based inference in phylogenetics [47], which may be a tractable approach also for ABC-based methods. Yet an adequate model for a complex system is very likely to require intensive computation irrespectively of the chosen method of inference, and it is up to the user to select a method that is suitable for the particular application in question.

Curse-of-dimensionality. High-dimensional datasets and high-dimensional parameter spaces can require an extremely large number of parameter points to be simulated in ABC-based studies to obtain a reasonable level of accuracy for the posterior inferences. In such situations, the computational cost is severely increased and may in the worst case render the computational analysis intractable. These are examples of well-known phenomena, which are usually referred to with the umbrella term curse-ofdimensionality [48].

To assess how severely the dimensionality of a dataset affects the analysis within the context of $\mathrm{ABC}$, analytical formulas have been derived for the error of the $\mathrm{ABC}$ estimators as functions of the dimension of the summary statistics [49-50]. In addition, Blum and François have investigated how the dimension of the summary statistics is related to the mean squared error for different correction adjustments to the error of $\mathrm{ABC}$ estimators. It was also argued that dimension reduction techniques are useful to avoid the curse-of-dimensionality, due to a potentially lower dimensional underlying structure of summary statistics [49].
Motivated by minimizing the quadratic loss of $\mathrm{ABC}$ estimators, Fearnhead and Prangle have proposed a scheme to project (possibly high-dimensional) data into estimates of the parameter posterior means; these means, now having the same dimension as the parameters, are then used as summary statistics for ABC [50].

$\mathrm{ABC}$ can be used to infer problems in high-dimensional parameter spaces, although one should account for the possibility of overfitting (e.g., see the model selection methods in [39-40]). However, the probability of accepting the simulated values for the parameters under a given tolerance with the $\mathrm{ABC}$ rejection algorithm typically decreases exponentially with increasing dimensionality of the parameter space (due to the global acceptance criterion) [1 1]. Although no computational method (based on ABC or not) seems to be able to break the curse-of-dimensionality, methods have recently been developed to handle high-dimensional parameter spaces under certain assumptions (e.g., based on polynomial approximation on sparse grids [51], which could potentially heavily reduce the simulation times for $\mathrm{ABC}$ ). However, the applicability of such methods is problem dependent, and the difficulty of exploring parameter spaces should in general not be underestimated. For example, the introduction of deterministic global parameter estimation led to reports that the global optima obtained in several previous studies of low-dimensional problems were incorrect [52]. For certain problems, it might therefore be difficult to know whether the model is incorrect or, as discussed above, whether the explored region of the parameter space is inappropriate [21]. A more pragmatic approach is to cut the scope of the problem through model reduction [11].

\section{Software for Application of ABC}

A number of software packages are currently available for application of ABC to particular classes of statistical models. An assortment of ABC-based software is presented in Table 3.

The suitability of individual software packages is dependent on the specific application at hand, the computer system environment, and the algorithms required.

\section{Conclusion}

In conclusion, $\mathrm{ABC}$ represents a class of well-founded and powerful methods for Bayesian statistical inference. However, reliable application of $\mathrm{ABC}$ requires additional caution to be considered, due to the approximations and biases introduced at the different stages of the approach. In its current incarnation, the $\mathrm{ABC}$ toolkit as a whole is best suited for inference about parameters or predictive inferences about observables in the presence of a single or few candidate model(s). How to make ABC practically feasible for problems involving large sets of models and/or high-dimensional target parameter spaces is currently largely an open issue. Since the computation of the likelihood function is bypassed, it can be tempting to attack high-dimensional problems using ABC, but inevitably this comes bundled with new challenges that investigators need to be aware of at each step of their analyses.

\section{Supporting Information}

Text S1 Version history of the text file (XML). (XML)

Text S2 Peer reviews and response to reviews. Human-readable versions of the reviews and authors' responses are available as comments on this article.

(XML) 


\section{Acknowledgments}

We would like to thank Matthew Astley, Joachim M. Buhmann, Sotiris Dimopoulos, Nick Goldman, Darren Logan, Joerg Stelling, and Elias Zamora-Sillero for useful discussions and/or feedback on the manuscript. We also thank Christian P. Robert and Dennis Prangle for their constructive referee reports and Daniel Mietchen for editorial work and

\section{References}

1. Rubin DB (1984) Bayesianly justifiable and relevant frequency calculations for the applies statistician. The Annals of Statistics 12: 1151-1172.

2. Diggle PJ, Gratton J (1984) Monte Carlo methods of inference for implicit statistical models. Journal of the Royal Statistical Society, Series B 46: 193-227.

3. Bartlett MS (1963) The spectral analysis of point processes. Journal of the Royal Statistical Society, Series B 25: 264-296

4. Hoel DG, Mitchell TJ (1971) The simulation, fitting and testing of a stochastic cellular proliferation model. Biometrics 27: 191-199.

5. Tavare S, Balding DJ, Griffiths RC, Donnelly P (1997) Inferring coalescence times from DNA sequence data. Genetics 145: 505-518.

6. Pritchard JK, Seielstad MT, Perez-Lezaun A, Feldman MW (1999) Population growth of human $\mathrm{Y}$ chromosomes: a study of $\mathrm{Y}$ chromosome microsatellites. Molecular Biology and Evolution 16: 1791-1798.

7. Beaumont MA, Zhang W, Balding DJ (2002) Approximate Bayesian computation in population genetics. Genetics 162: 2025-2035

8. Busetto AG, Buhmann J (2009) Stable Bayesian parameter estimation for biological dynamical systems. IEEE Computer Society Press, Proceedings IEEE CSE'09: 148-157.

9. Beaumont MA (2010) Approximate Bayesian computation in evolution and ecology. Annual Review of Ecology, Evolution, and Systematics 41: 379-406.

10. Bertorelle G, Benazzo A, Mona S (2010) ABC as a flexible framework to estimate demography over space and time: some cons, many pros. Molecular Ecology 19: 2609-2625.

11. Csilléry K, Blum MGB, Gaggiotti OE, François O (2010) Approximate Bayesian Computation $(\mathrm{ABC})$ in practice. Trends in Ecology \& Evolution 25: 410-418.

12. Didelot X, Everitt RG, Johansen AM, Lawson DJ (2011) Likelihood-free estimation of model evidence. Bayesian Analysis 6: 49-76.

13. Lai K, Robertson MJ, Schaffer DV (2004) The sonic hedgehog signaling system as a bistable genetic switch. Biophys J 86: 2748-2757.

14. Marin JM, Pudlo P, Robert CP, Ryder RJ (2011) Approximate Bayesian computational methods. Statistics and Computing 22: 1167-1180.

15. Wilkinson RG (2007). Bayesian estimation of primate divergence times. $\mathrm{PhD}$ thesis, University of Cambridge.

16. Grelaud A, Marin JM, Robert C, Rodolphe F, Tally F (2009) Likelihood-free methods for model choice in Gibbs random fields. Bayesian Analysis 3: 427-442.

17. Toni T, Stumpf MPH (2010) Simulation-based model selection for dynamical systems in systems and population biology. Bioinformatics 26: 104-110.

18. Robert CP, Cornuet J-M, Marin J-M, Pillai NS (2011) Lack of confidence in approximate Bayesian computation model choice. Proc Natl Acad Sci USA 108: $15112-15117$

19. Templeton AR (2009) Why does a method that fails continue to be used? The answer. Evolution 63: 807-812.

20. Templeton AR (2008) Nested clade analysis: an extensively validated method for strong phylogeographic inference. Molecular Ecology 17: 1877-1880.

21. Templeton AR (2009) Statistical hypothesis testing in intraspecific phylogeography: nested clade phylogeographical analysis vs. approximate Bayesian computation. Molecular Ecology 18: 319-331.

22. Berger JO, Fienberg SE, Raftery AE, Robert CP (2010) Incoherent phylogeographic inference. Proc Natl Acad Sci USA 107: E157-E157.

23. Sisson SA, Fan Y, Tanaka MM (2007) Sequential Monte Carlo without likelihoods. Proc Natl Acad Sci USA 104: 1760-1765.

24. Dean TA, Singh SS, Jasra A, Peters GW (2011) Parameter estimation for hidden Markov models with intractable likelihoods. arXiv: $11035399 \mathrm{v} 1$. Available: http://arxiv.org/abs/1103.5399.

25. Fearnhead P, Prangle D (2011) Constructing summary statistics for approximate Bayesian Computation: semi-automatic ABC. arXiv:10041112v2. Available: http://arxiv.org/abs/1004.1112.

26. Blum M, Francois O (2010) Non-linear regression models for approximate Bayesian computation. Stat Comp 20: 63-73.

27. Leuenberger C, Wegmann D (2009) Bayesian Computation and model selection without likelihoods. Genetics 184: 243-252.

28. Wilkinson RD (2009) Approximate Bayesian computation (ABC) gives exact results under the assumption of model error. arXiv:0811.3355. Available: http://arxiv.org/abs/0811.3355.

29. Blum MGB, Nunes MA, Prangle D, Sisson SA (2012) A comparative review of dimension reduction methods in approximate Bayesian computation. arxiv.org/ abs/1202.3819. Available: http://arxiv.org/abs/1202.3819.

30. Nunes MA, Balding DJ (2010) On optimal selection of summary statistics for approximate Bayesian computation. Stat Appl Genet Mol Biol 9: Article 34.

31. Joyce P, Marjoram P (2008) Approximately sufficient statistics and Bayesian computation. Stat Appl Genet Mol Biol 7: Article 26. help to bring the article up to Wikipedia standards. This article started as assignment for the graduate course "Reviews in Computational Biology" (263-5151-00L) at ETH Zurich. The version history of the text file and the peer reviews (and response to reviews) are available as supporting information in Text S1 and S2.

32. Wegmann D, Leuenberger C, Excoffier L (2009) Efficient approximate Bayesian computation coupled with Markov chain Monte Carlo without likelihood. Genetics 182: 1207-1218.

33. Marjoram P, Molitor J, Plagnol V, Tavare S (2003) Markov chain Monte Carlo without likelihoods. Proc Natl Acad Sci USA 100: 15324-15328.

34. Marin J-M, Pillai NS, Robert CP, Rosseau J (2011) Relevant statistics for Bayesian model choice. arXiv:11104700v1. Available: http://arxiv.org/abs/ $1110.4700 \mathrm{v} 1$.

35. Toni T, Welch D, Strelkowa N, Ipsen A, Stumpf M (2007) Approximate Bayesian computation scheme for parameter inference and model selection in dynamical systems. J R Soc Interface 6: 187-202.

36. Arlot S, Celisse A (2010) A survey of cross-validation procedures for model selection. Statistical Surveys 4: 40-79.

37. Dawid A (1984) Present position and potential developments: some personal views. Statistical theory: the prequential approach. Journal of the Royal Statistical Society Series A: 278-292.

38. Vehtari A, Lampinen J (2002) Bayesian model assessment and comparison using cross-validation predictive densities. Neural Computation 14: 2439-2468.

39. Ratmann O, Andrieu C, Wiuf C, Richardson S (2009) Model criticism based on likelihood-free inference, with an application to protein network evolution. Proc Natl Acad Sci USA 106: 10576-10581.

40. Francois O, Laval G (2011) Deviance information criteria for model selection in approximate Bayesian computation. Stat Appl Genet Mol Biol 10: Article 33.

41. Templeton AR (2010) Coherent and incoherent inference in phylogeography and human evolution. Proc Natl Acad Sci USA 107: 6376-6381.

42. Beaumont MA, Nielsen R, Robert C, Hey J, Gaggiotti O, et al. (2010) In defence of model-based inference in phylogeography. Molecular Ecology 19: $436-446$.

43. Jaynes ET (1968) Prior probabilities. IEEE Transactions on Systems Science and Cybernetics 4: 227-241.

44. Berger JO (2006) The case for objective Bayesian analysis. Bayesian Analysis 1: 385-402.

45. Beaumont MA, Cornuet J-M, Marin J-M, Robert CP (2009) Adaptive approximate Bayesian computation. Biometrika 96: 983-990.

46. Del Moral P, Doucet A, Jasra A (2012) An adaptive sequential Monte Carlo method for approximate Bayesian computation. Statistics and Computing 22: $1009-1020$

47. Feng X, Buell DA, Rose JR, Waddellb PJ (2003) Parallel algorithms for Bayesian phylogenetic inference. Journal of Parallel and Distributed Computing 63: 707718 .

48. Bellman R (1961) Adaptive control processes: a guided tour. Princeton, NJ: Princeton University Press. $255 \mathrm{pp}$

49. Blum MGB (2010) Approximate Bayesian Computation: a nonparametric perspective. Journal of the American Statistical Association 105: 1178-1187.

50. Fearnhead P, Prangle D (2012) Constructing summary statistics for approximate Bayesian computation: semi-automatic approximate Bayesian computation. Journal of the Royal Statistical Society Series B 74: 419-474.

51. Gerstner T, Griebel M (2003) Dimension-adaptive tensor-product quadrature. Computing 71: 65-87.

52. Singer AB, Taylor JW, Barton PI, Green WH (2006) Global dynamic optimization for parameter estimation in chemical kinetics. J Phys Chem A 110: 971-976.

53. Cornuet J-M, Santos F, Beaumont M, Robert CP, Marin J-M, et al. (2008) Inferring population history with DIY ABC: a user-friendly approach to approximate Bayesian computation. Bioinformatics 24: 2713-2719.

54. Csilléry K, François O, Blum MGB (2012) ABC: an R package for approximate Bayesian computation (ABC). Methods in Ecology and Evolution 3: 475-479.

55. Liepe J, Barnes C, Cule E, Erguler K, Kirk P, Toni T, Stumpf MP (2010) ABCSysBio-approximate Bayesian computation in Python with GPU support. Bioinformatics 26: 1797-1799.

56. Wegmann D, Leuenberger C, Neuenschwander S, Excoffier L (2010) ABCtoolbox: a versatile toolkit for approximate Bayesian computations. BMC Bioinformatics 11: 116 .

57. Hickerson MJ, Stahl E, Takebayashi N (2007) msBayes: pipeline for testing comparative phylogeographic histories using hierarchical approximate Bayesian computation, BMC Bioinformatics 8(268): 1471-2105.

58. Lopes JS, Balding D, Beaumont MA (2009) PopABC: a program to infer historical demographic parameters. Bioinformatics 25: 2747-2749.

59. Tallmon DA, Koyuk A, Luikart G, Beaumont MA (2008) COMPUTER PROGRAMS: onesamp: a program to estimate effective population size using approximate Bayesian computation. Molecular Ecology Resources 8: 299-301. 
60. Foll M, Baumont MA, Gaggiotti OE (2008) An Approximate Bayesian Computation approach to overcome biases that arise when using AFLP markers to study population structure. Genetics 179: 927-939.
61. Bray TC, Sousa VC, Parreira B, Bruford MW, Chikhi L (2010) 2BAD: an application to estimate the parental contributions during two independent admisture events. Molecular Ecology Resources 10: 538-541. 